\title{
Efficiency at maximum power output of quantum heat engines under finite-time operation
}

Jianhui Wang* and Jizhou He

Department of Physics, Nanchang University, Nanchang 330031, China

Zhaoqi $\mathrm{Wu}$

Department of Mathematics, Nanchang University, Nanchang 330031, China

\begin{abstract}
We study the efficiency at maximum power, $\eta_{m}$, of irreversible quantum Carnot engines (QCEs) that perform finite-time cycles between a hot and a cold reservoir at temperatures $T_{h}$ and $T_{c}$, respectively. For QCEs in the reversible limit (long cycle period, zero dissipation), $\eta_{m}$ becomes identical to Carnot efficiency $\eta_{C}=1-\frac{T_{c}}{T_{h}}$. For QCE cycles in which nonadiabatic dissipation and time spent on two adiabats are included, the efficiency $\eta_{m}$ at maximum power output is bounded from above by $\frac{\eta_{C}}{2-\eta_{C}}$ and from below by $\frac{\eta_{C}}{2}$. In the case of symmetric dissipation, the CurzonAhlborn efficiency $\eta_{C A}=1-\sqrt{\frac{T_{c}}{T_{h}}}$ is recovered under the condition that the time allocation between the adiabats and the contact time with the reservoir satisfy a certain relation.

Keywords: heat engine, finite-time cycle, nonadiabatic dissipation.

PACS number(s): 05.70.Ln, 05.30.-d
\end{abstract}

*Electronic address: physwjh@gmail.com 


\section{INTRODUCTION}

The concept of Carnot efficiency is of paramount importance in thermodynamics, since the Carnot cycle is the most efficient heat engine cycle allowed by physical laws. When the thermodynamic second law states that not all the supplied heat is applied to producing work, the Carnot efficiency presents the limiting value on the fraction of the heat which can be so used. Although the quasistatic Carnot cycle has the highest efficiency, it outputs zero power because it takes infinite time to output a finite amount of work. By contrast, Curzon and Ahlborn [1] considered a finite-time Carnot cycle under the assumption of endoreversibility that irreversible processes occur only through these heat exchanges, they obtained the efficiency $\eta_{C A}$ at maximum power output as

$$
\eta_{C A}=1-\sqrt{\frac{T_{c}}{T_{h}}}
$$

where $T_{h}$ and $T_{c}$ are the temperatures of the hot and cold heat reservoirs, respectively. The Curzon-Ahlborn (CA) paper has triggered the development of research into finite time thermodynamics [2 19]. The above $\eta_{c \wedge}$ is usually called the CA efficiency, describing the efficiency of several engine models [2, 16, 18, 20] and of actual thermal plants [1, 2, 6 6 ,, 10$]$ very well. The CA efficiency has been found to be a universal result in the case of the low, asymmetric dissipation, by optimizing power output with respect to time allocation when time durations in adiabats and nonadiabatic phenomenon were ignored [8].

Great efforts have been devoted to the study of quantum heat engines [4, 5, 10 12, 21 29], beginning with the concept of quantum heat engine introduced by Scovil and Schulz-DuBois [30]. Quantum heat engines differ from classical counterparts mainly in the following three respects: (i) the working substance is composed of quantum matter such as spin systems 44, 5, 10, 11, 26], harmonic oscillator systems [5, 10, 12], two-level or multilevel systems [21 24], cavity quantum electrodynamics systems [24, 27-29], etc. (ii) The state of the system is depicted by a quantum-mechanical operator, and the thermodynamic observables are associated with the expectation of values of operators [10 12]. (iii) Quantum equations of motion are used to describe the time evolution of the observables in quantum heat engines, which can avoid using phenomenological heat transfer laws [10 12].

The previous literature discussed the heat engine models in the sudden limit in which the adiabatic process is a spontaneous switching and thus the time allocation on adiabats 
is negligible [8, 16, 17]. Thus it is significant to study more general models in which the "adiabatic" process ( we take the two corresponding processes as two quantum "adiabats" throughout the paper) takes finite time as well become nonadiabatic [31]. During a quantum adiabatic process, the variation of the eigenspectrum (quantum state) of the system must be so slow that the quantum adiabatic theorem [22 24, 32] can apply. Otherwise, nonadiabatic dissipation (e.g., inner friction [11, 12, 26]) occurs because of rapid change in the energy level structure of the quantum system. Particularly nonadiabatic dissipation has been found to have a profound influence on the performance of quantum heat engines [11, 12, 26]. Including nonadiabatic dissipation is therefore essential for more realistic models of quantum heat engines.

In this paper, we study the efficiency at maximum power output of QCEs performing finite time cycles, in which the time of any adiabat and nonadiabatic dissipation are considered. We assume that the external parameter affecting the energy spectrum varies at a small but fixed speed which, however, may not be slow enough and thus to cause nonadiabatic phenomenon. We derive the cycle period that consists of times spent both on the two quantum isotherms and on the two quantum adiabats. We show that the efficiency at maximum power output converges to an upper and a lower bound in the limits of extremely asymmetric dissipation. Based on the low-dissipation assumption that the irreversible entropy production in a thermodynamic process is inversely proportional to the time required to complete that process, our approach similar to that of the classical thermodynamics predicts that the CA efficiency turns out to be an exact and universal property for QCEs operating under the conditions that the dissipation is symmetric and the time allocation between the adiabats and the contact time with the reservoir satisfy a certain relation.

\section{EFFICIENCY AT MAXIMUM POWER OUTPUT}

We consider a quantum system whose Schrödinger's equation is given by $H\left|u_{n}\right\rangle=E_{n}\left|u_{n}\right\rangle$, where $H,\left|u_{n}\right\rangle$ and $E_{n}$ are the Hamiltonian of the system, its $n$th eigenstate and eigenenergy, respectively. The internal energy $U$ reads $U=\sum_{n} E_{n} P_{n}$, where $P_{n}$ is the mean occupation probability of the $n$th eigenstate and obeys the canonical distribution $P_{n}=\frac{1}{Z} e^{-E_{n} / k_{B} T}$ in equilibrium, with the canonical partition function $Z=\sum_{n} e^{-E_{n} / k_{B} T}$. Derivation of $U$ leads to the first quantum thermodynamic law $d U=\sum_{n} E_{n} d P_{n}+\sum_{n} P_{n} d E_{n}$. Analogous to the 
classical thermodynamic first law, the first law of thermodynamics in quantum-mechanical systems is [23-25] $d U=đ Q+d W$, in which $d Q=\sum_{n} E_{n} d P_{n}$ and $d W=\sum_{n} P_{n} d E_{n}$ depict the heat exchange and work done, respectively, during a thermodynamic process. Note that $\sum_{n} E_{n} d P_{n}$ is associated with the heat exchange because $₫ Q=T d S$ with the entropy $S=-k_{B} P_{n} \ln P_{n}$. Motivated by the definition of the generalized force $F$ for a classical system, we define analogously the force for a quantum system as $F=\sum_{n} P_{n} \frac{\partial E_{n}(X)}{\partial X}$, where $X$ is the external parameter (generalized coordinate corresponding to the force $F$ ) $[23-$ 25, 33]. Here the force $F$ and generalized coordinate $X$ are state variables [25, 33] and quantum versions of the classical pressure $P_{r}$ and volume $V$, respectively.
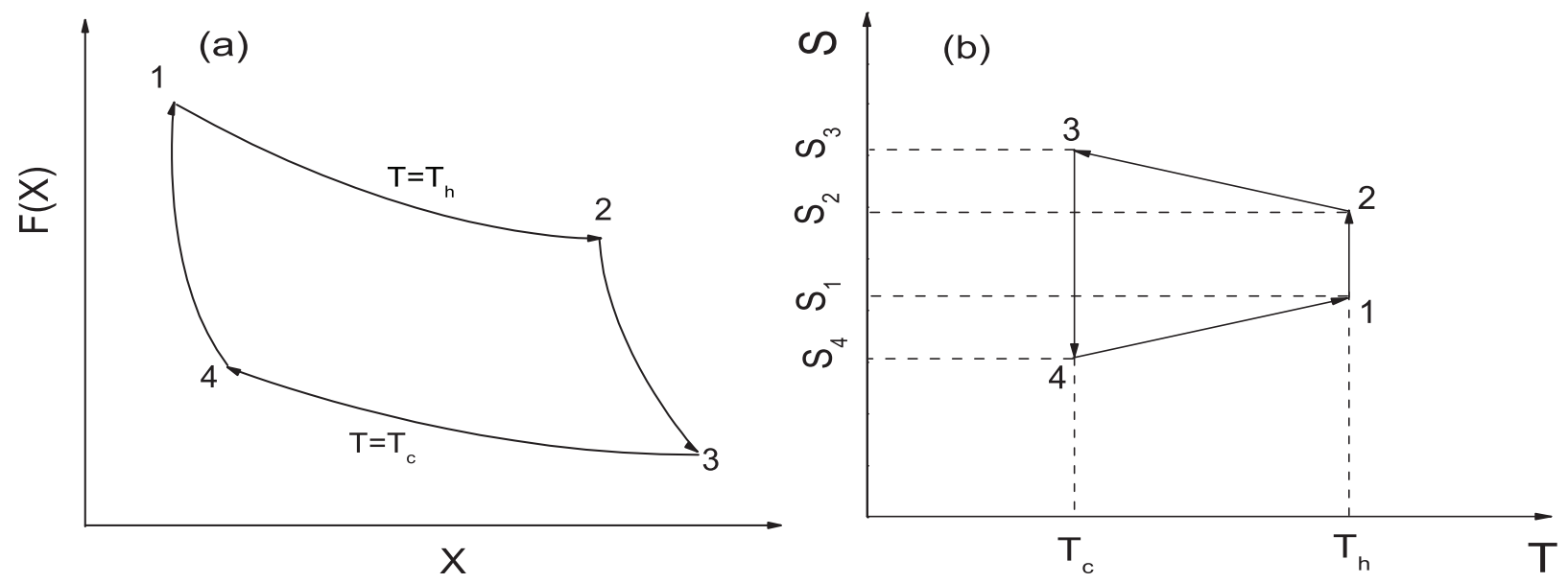

FIG. 1: Schematic diagram of an irreversible QCE cycle in the plane of the external parameter $X$ and force $F(X)$ (a) and of the Temperature $T$ and entropy $S$ (b). The values of the external parameter $X$ and of the entropy $S$ at the four special instants are indicated.

The generalized force $F(X)$ alters the generalized coordinate $X$ that affects the eigenspectrum of the system during a thermodynamic process. The quantum Carnot cycle $1 \rightarrow 2 \rightarrow 3 \rightarrow 4 \rightarrow 1$ is drawn in the $(F, X)$ plane, which is the quantum version of the classical $\left(P_{r}, V\right)$ plane (See. Fig. 1a). During two quantum isothermal processes $1 \rightarrow 2$ and $3 \rightarrow 4$, the working substance is coupled to a hot and a cold heat reservoir at constant temperatures $T_{h}$ and $T_{c}$, respectively. We apply $₫ Q=T d S$ directly to the calculation of the heat exchange $d Q$ in any quantum isothermal process. Let $S\left(X_{i}\right)$ and $X_{i}$ be the entropies and the external parameters at the instants $i$ with $i=1,2,3,4$, the heat amount $Q_{h}$ absorbed from the hot reservoir and the heat amount $Q_{c}$ released to the cold reservoir are, respectively, $Q_{h}=T_{h}\left[S\left(X_{2}\right)-S\left(X_{1}\right)\right]$ and $Q_{c}=T_{c}\left|\left[S\left(X_{4}\right)-S\left(X_{3}\right)\right]\right|$. When the Carnot 
cycle is reversible, the system couples to the heat reservoir for a sufficiently long time until the system remains infinitesimally close to equilibrium all along the cycle, and in order for the adiabatic theory to remain valid, the time scale of the change of the quantum state must be much larger than that of the dynamical one, $\sim E / \hbar[22,23$, 32]. For the reversible cycle where $S\left(X_{2}\right)=S\left(X_{3}\right)$ and $S\left(X_{1}\right)=S\left(X_{4}\right)$, we recover the Carnot efficiency $\eta_{C}=1-\frac{T_{c}}{T_{h}}$, which is independent of the properties of the quantum working substance. We denote by $t_{12}\left(t_{34}\right)$ the time durations during which the system is coupled to the hot (cold) reservoir along a cycle. In the branch $2 \rightarrow 3(4 \rightarrow 1)$, the working substance is decoupled from the hot (cold) reservoir, and the entropy changes from $S_{2}$ to $S_{3}\left(S_{4}\right.$ to $\left.S_{1}\right)$ during a period $t_{23}$ ( $\left.t_{41}\right)$, as shown in Fig. 1 $1 \mathrm{~b}$, where $S_{i} \equiv S\left(X_{i}\right)$ with $i=1,2,3,4$.

Let us consider a QCE under finite-time operation. Finite-time cycles move the system away from the equilibrium, leading to irreversibility of the engine. Although the system needs no close to equilibrium during the isothermal process, the system remains in an equilibrium state with the heat reservoir at special instants $i$ with $i=1,2,3,4$. Under such a circumstance, the thermodynamic quantities of the system-in particular the entropy-are well defined at these instants. During the processes $2 \rightarrow 3$ and $4 \rightarrow 1$, the rapid change (compared with time scale, $\sim E / \hbar)$ in the energy level structure of the system results in quantum nonadiabatic phenomenon. We emphasize that in any quantum thermodynamic process in which the low dissipation exists the system relaxation is assumed to be fast compared to the time of the process [8]. During a quantum isothermal (adiabatic) process, the entropy production caused by weak dissipation can be written as $\Sigma_{h} / t_{12}$ or $\Sigma_{c} / t_{34}\left(\Sigma_{a} / t_{23}\right.$ or $\left.\Sigma_{a} / t_{41}\right)$, since the reversible regime is approached in the infinite time durations. Thus, the real heat exchanges $Q_{h}$ and $Q_{c}$ are $Q_{h}=T_{h} \Delta S-T_{h} \frac{\Sigma_{h}}{t_{12}}$ and $Q_{c}=T_{c} \Delta S+T_{c} \frac{\Sigma_{c}}{t_{34}}+T_{c}\left(\frac{\Sigma_{a}}{t_{23}}+\frac{\Sigma_{a}}{t_{41}}\right)$, with $\Delta S=S\left(X_{2}\right)-S\left(X_{1}\right)=\left|\left[S\left(X_{4}\right)-S\left(X_{3}\right)\right]\right|-\left(\frac{\Sigma_{a}}{t_{23}}+\frac{\Sigma_{a}}{t_{41}}\right)$. According to the first law of the thermodynamics, the work $W$ per cycle is determined by

$$
W=Q_{h}-Q_{c}=\left(T_{h}-T_{c}\right) \Delta S-T_{h} \frac{\Sigma_{h}}{t_{12}}-T_{c} \frac{\Sigma_{c}}{t_{34}}-T_{c}\left(\frac{\Sigma_{a}}{t_{23}}+\frac{\Sigma_{a}}{t_{41}}\right) .
$$

Let $v(t)$ and $\tau$ be the speed of the change of $X$ and the cycle period, respectively. Then we have

$$
\begin{aligned}
X_{0} & =\left(X_{2}-X_{1}\right)+\left(X_{3}-X_{2}\right)+\left(X_{3}-X_{4}\right)+\left(X_{4}-X_{1}\right) \\
& =2\left(X_{3}-X_{1}\right)=\int_{0}^{\tau} v(t) d t=\bar{v} \tau
\end{aligned}
$$


where $\bar{v}$ is the average speed of the change of $X$. The displacement of $X$ after a single cycle is zero and thus $X$ is a state variable, though the total change per cycle $X_{0}$ is not equal to zero. The times spent on two isothermal processes can be expressed as $t_{12}=\left(X_{2}-X_{1}\right) / \bar{v}$ and $t_{34}=\left(X_{3}-X_{4}\right) / \bar{v}$, respectively, while the times of two adiabats are $t_{23}=\left(X_{3}-X_{2}\right) / \bar{v}$ and $t_{41}=\left(X_{4}-X_{1}\right) / \bar{v}$. Therefore, the power output $P=W / \tau$ and the efficiency $\eta=W / Q_{h}$ are

$$
P=\frac{\bar{v}}{2\left(X_{3}-X_{1}\right)}\left[\left(T_{h}-T_{c}\right) \Delta S-\frac{\bar{v} T_{h} \Sigma_{h}}{\left(X_{2}-X_{1}\right)}-\frac{\bar{v} T_{c} \Sigma_{c}}{\left(X_{3}-X_{4}\right)}-\frac{\bar{v} T_{c} \Sigma_{a}}{\left(X_{3}-X_{2}\right)}-\frac{\bar{v} T_{c} \Sigma_{a}}{\left(X_{4}-X_{1}\right)}\right]
$$

and

$$
\eta=\frac{\left(T_{h}-T_{c}\right) \Delta S-\frac{\bar{v} T_{h} \Sigma_{h}}{\left(X_{2}-X_{1}\right)}-\frac{\bar{v} T_{c} \Sigma_{c}}{\left(X_{3}-X_{4}\right)}-\frac{\bar{v} T_{c} \Sigma_{a}}{\left(X_{3}-X_{2}\right)}-\frac{\bar{v} T_{c} \Sigma_{a}}{\left(X_{4}-X_{1}\right)}}{T_{h} \Delta S-\frac{\bar{v} T_{h} \Sigma_{h}}{\left(X_{2}-X_{1}\right)}}
$$

respectively. Here $\Delta S$ is a state variable determined only by the initial and final states of the isothermal process. The generalized coordinates $X_{i}$ with $i=1,2,3,4$, corresponding to the system volume $V$ in the classical thermodynamics, are state variables and independent of the detailed protocols. To specify the time allocation at maximum power output, the values of $X_{i}$ as well as the average speed $\bar{v}$ should be optimized. We will the optimize power output $P$ over the average speed $\bar{v}$ and the variables $X_{i}$ to obtain the time allocation during a cycle and thus to determine the corresponding efficiency. We will assume, for simplicity, that the initial value of the external parameter is a constant, i.e, $X_{1}=X_{1}^{0}$. The maximum power is therefore found by setting the derivatives of $P$ with respect to the average speed $\bar{v}$ and $X_{i}$ with $i=2,3,4$ equal to zero.

The maximization conditions $\left.\frac{\partial P}{\partial X_{i}}\right|_{X_{i}=X_{i}^{m}}=0$ and $\left.\frac{\partial P}{\partial \bar{v}}\right|_{\bar{v}=\bar{v}_{m}}=0$ give the physical solution. The value of $X_{3}^{m}$ is determined by the following equation

$$
\begin{aligned}
\left(T_{h}-T_{c}\right) \Delta S & =\bar{v}_{m} T_{c} \Sigma_{a}\left(\frac{1}{X_{3}^{m}-X_{2}^{m}}+\frac{1}{X_{4}^{m}-X_{1}^{m}}\right)+\frac{\bar{v}_{m} T_{c} \Sigma_{c}}{X_{3}^{m}-X_{4}^{m}}+\bar{v}_{m} T_{c}\left(X_{3}^{m}-X_{1}^{0}\right) \\
& \times\left[\frac{\Sigma_{a}}{\left(X_{3}^{m}-X_{2}^{m}\right)^{2}}+\frac{\Sigma_{c}}{\left(X_{3}^{m}-X_{4}^{m}\right)^{2}}\right]+\frac{\bar{v}_{m} T_{h} \Sigma_{h}}{X_{2}^{m}-X_{1}^{0}}
\end{aligned}
$$

where

$$
\begin{gathered}
\bar{v}_{m}=\frac{1}{2} \frac{\left(T_{h}-T_{c}\right) \Delta S}{\left(\frac{1}{X_{3}^{m}-X_{2}^{m}}+\frac{1}{X_{4}^{m}-X_{1}^{0}}\right) T_{c} \Sigma_{a}+\frac{1}{X_{3}^{m}-X_{4}^{m}} T_{c} \Sigma_{c}+\frac{1}{X_{2}^{m}-X_{1}^{0}} T_{h} \Sigma_{h}}, \\
X_{2}^{m}=\frac{\left|T_{h} \Sigma_{h} X_{3}^{m}-T_{c} \Sigma_{a} X_{1}^{0}\right|+\left(X_{3}^{m}-X_{1}^{0}\right) \sqrt{T_{c} T_{h} \Sigma_{a} \Sigma_{h}}}{\left|T_{h} \Sigma_{h}-T_{c} \Sigma_{a}\right|},
\end{gathered}
$$

and

$$
X_{4}^{m}=\frac{\left|\Sigma_{a} X_{3}^{m}-\Sigma_{c} X_{1}^{0}\right|+\left(X_{3}^{m}-X_{1}^{0}\right) \sqrt{\Sigma_{a} \Sigma_{c}}}{\left|\Sigma_{a}-\Sigma_{c}\right|} .
$$


From Eqs. (6) and (7), we find that the optimal value of $X_{3}^{m}$ with fixed value of $X_{1}^{0}$ is independent of the value of the state variable $\Delta S$, as expected. Substitution of Eqs. (77), (8) and (9) into Eq. (6) leads to the fundamental optimal relationship between $X_{3}^{m}$ and $X_{1}^{0}$ at maximum power output. Under the assumption that the value of $X_{1}$ is fixed at the start of the engine cycle, Eq. (6) can be done numerically for given values of entropy production $\Sigma_{a}, \Sigma_{c}$ and $\Sigma_{h}$ along the specific processes and of temperatures $T_{h}$ and $T_{c}$. Once we have obtained the optimal relationship between $X_{1}^{0}$ and $X_{3}^{m}$ at maximum power output $P$, we can then determine the optimal values of $X_{i}^{m}$ with $i=2,4$, and the average speed $\bar{v}_{m}$ by Eqs. (7), (8), and (91).

Substituting Eq. (17) into Eq. (5), we find the expression for the efficiency at maximum power as follows:

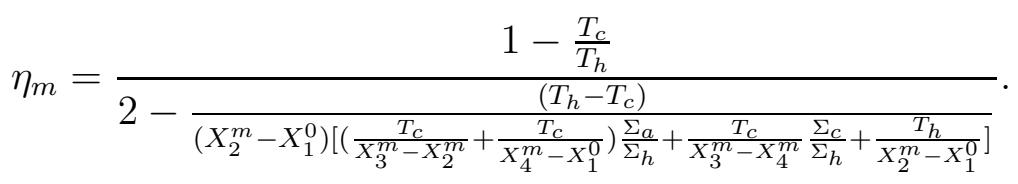

Eq. (10) together with Eqs. (6) , (7), (8)), and (9), as one of our main results, conveys the following physical features:

(i) The nonadiabatic dissipation is neglected, i.e., $\Sigma_{a} \rightarrow 0$. In such a case, the limits $\frac{\Sigma_{c}}{\Sigma_{h}} \rightarrow 0$ and $\frac{\Sigma_{c}}{\Sigma_{h}} \rightarrow \infty$, lead to the result that the efficiency $\eta_{m}$ at the maximum power approaches to the upper bound $\eta_{+} \equiv \frac{\eta_{C}}{2-\eta_{C}}$ and to the lower bound $\eta_{-} \equiv \frac{\eta_{C}}{2}$, respectively. That is, the efficiency $\eta_{m}$ at the maximum power satisfies the following condition:

$$
\frac{\eta_{C}}{2} \equiv \eta_{-} \leq \eta_{m} \leq \eta_{+} \equiv \frac{\eta_{C}}{2-\eta_{C}}
$$

In Fig. 2 we plot the efficiency (10) as a function of $\eta_{C}$ comparing $\eta_{C A}$ with the upper and lower bounds (11). The lower and upper bounds, which are reached in the completely asymmetric limits $\frac{\Sigma_{c}}{\Sigma_{h}} \rightarrow 0$ and $\frac{\Sigma_{c}}{\Sigma_{h}} \rightarrow \infty$, are identical to the corresponding those derived in different approaches [8]. However, unlike the previous literature in which the times of two adiabats are ignored, the times spent on the two adiabats in the quantum Carnot cycle are taken into account. If the symmetric dissipation $\Sigma_{c}=\Sigma_{h}$ and $\frac{X_{2}^{m}-X_{1}^{0}}{X_{3}^{m}-X_{4}^{m}}=\sqrt{\frac{T_{h}}{T_{c}}}$, i.e., the time allocation to the hot and cold processes at maximum power:

$$
\frac{t_{12}}{t_{34}}=\sqrt{\frac{T_{h}}{T_{c}}}
$$

we can recover the CA efficiency $\eta_{m}=\eta_{C A}=1-\sqrt{\frac{T_{c}}{T_{h}}}$ by using Eq. (10). Result of 
consideration of symmetric dissipation agrees with that obtained by optimizing power output with respect to the times of the two isothermal processes [8, 34].

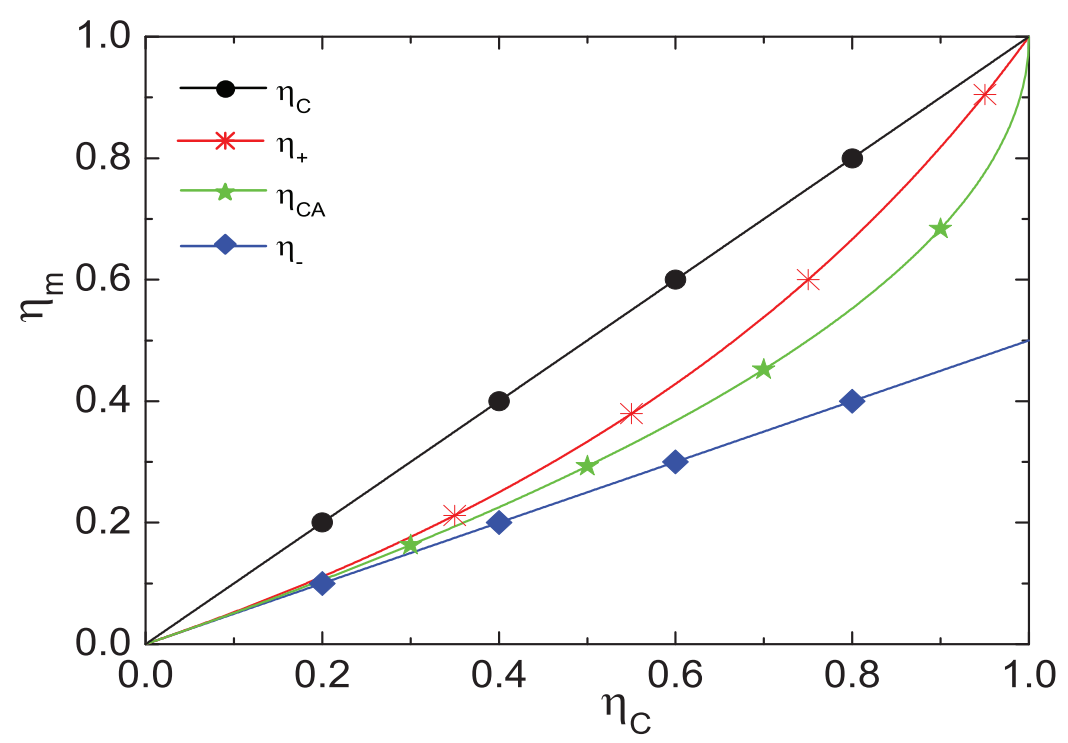

FIG. 2: (Color online). Efficiency $\eta_{m}$ at maximum power as a function of the Carnot value $\eta_{C}$. The upper and lower bounds of the efficiency, $\eta_{+}$and $\eta_{-}$given in Eq. (11), are represented by a red and a blue solid line with starts and squares, respectively. The CA efficiency $\eta_{C A}$ is denoted by a green solid line with 5-pointed stars, while the Carnot efficiency $\eta_{C}$ by a black solid line with circles.

(ii) There is nonadiabatic dissipation, while the dissipation in at least one quantum isothermal process is not considered, namely, $\Sigma_{a} \neq 0$, and $\Sigma_{h} \rightarrow 0$ (or $\Sigma_{c} \rightarrow 0$ ). From Eq. (10) we find in this case

$$
\eta_{m}=\eta_{-}=\frac{\eta_{C}}{2}
$$

which is independent of the values of both $\Sigma_{a}$ and $\Sigma_{c}\left(\right.$ or $\left.\Sigma_{h}\right)$. The lower bound, which is found in the case when nonadiabatic dissipation exists but dissipation vanishes in at least one isotherm, is particularly interesting. Although it coincides with a reported universal lower bound in Refs. [8, 17], it is derived in the generalized engine model with nonadiabatic phenomenon. Physically, even in the case when the two isothermal processes are reversible, inclusion of an arbitrary low dissipation in the adiabats reduces the efficiency at maximum power output to half the Carnot value $\frac{\eta_{C}}{2}$.

(iii) Dissipations in four quantum thermodynamic processes are equal, i.e., $\Sigma_{c} / \Sigma_{h}=1$ and $\Sigma_{a} / \Sigma_{h}=1$. Let $R_{x} \equiv\left(X_{2}^{m}-X_{1}^{0}\right)\left(\frac{1}{X_{3}^{m}-X_{2}^{m}}+\frac{1}{X_{4}^{m}-X_{1}^{0}}+\frac{1}{X_{3}^{m}-X_{4}^{m}}\right)$, in the limits $R_{x} \rightarrow 0$ 
and $R_{x} \rightarrow \infty$, the efficiency $\eta_{m}$ corresponding to maximum power output converges to the upper bound $\eta_{+}=\frac{\eta_{C}}{2-\eta_{C}}$ and to the lower bound $\eta_{-}=\frac{\eta_{C}}{2}$, respectively. Here the lower and upper bounds are equal to the corresponding those in previous studies, but extended to the irreversible QCEs in which the time spent on two adiabats and nonadiabatic dissipation are considered. According to Eq. (10), the CA efficiency $\eta_{C A}=1-\sqrt{\frac{T_{c}}{T_{h}}}$ is achieved when the times spent on the four quantum thermodynamic processes are distributed in such a way that

$$
\frac{t_{12}\left(\tau-t_{12}\right)}{t_{23} t_{34} t_{41}}=\sqrt{\frac{T_{h}}{T_{c}}},
$$

where $t_{12}=\left(X_{2}^{m}-X_{1}^{0}\right) / \bar{v}_{m}, t_{23}=\left(X_{3}^{m}-X_{2}^{m}\right) / \bar{v}_{m}, t_{34}=\left(X_{3}^{m}-X_{4}^{m}\right) / \bar{v}_{m}$, and $t_{41}=\left(X_{4}^{m}-\right.$ $\left.X_{1}^{0}\right) / \bar{v}_{m}$

\section{CONCLUSIONS}

In conclusion, we have determined efficiency at maximum power for a QCE engine performing finite time cycles. To correctly describe the irreversible QCEs the times spent on two adiabats and nonadiabatic phenomenon have been taken into account. In the limits of extremely asymmetric dissipation $\left(\frac{\Sigma_{c}}{\Sigma_{h}} \rightarrow 0\right.$ and $\frac{\Sigma_{c}}{\Sigma_{h}} \rightarrow \infty$, with $\left.\frac{\Sigma_{a}}{\Sigma_{h}} \rightarrow 0\right)$, the efficiency at maximum power output converges to an upper and a lower bound, coinciding with the result obtained previously in different approaches. When dissipation in any isothermal process vanishes, the efficiency at maximum power output is equal to the lower bound $\frac{\eta_{C}}{2}$. For the QCE with the symmetric dissipation but without nonadiabatic dissipation $\left(\frac{\Sigma_{c}}{\Sigma_{h}} \rightarrow 1\right.$, while $\frac{\Sigma_{a}}{\Sigma_{h}} \rightarrow 0$ ), we have derived CA efficiency at maximum power output, only provided that the ratio of the times of contact with two heat reservoirs satisfies the relation given as in Eq. (12). In the case of $\frac{\Sigma_{c}}{\Sigma_{h}} \rightarrow 1$ and $\frac{\Sigma_{a}}{\Sigma_{h}} \rightarrow 1$, we have also recovered CA efficiency at maximum power output, if the time allocations of four processes fulfill the condition in Eq. (14).

Acknowledgements: We gratefully acknowledge support for this work by the National Natural Science Foundation of China under Grant Nos. 11147200 and 11065008. J. H. Wang also gratefully acknowledges Z. C. Tu for his kind communications.

[1] F. Curzon and B. Ahlborn, Am. J. Phys. 43, 22 (1975). 
[2] M. Esposito, K. Lindenberg, and C. Van den Broeck, Europhys. Lett. 85, 60010 (2009); B. Rutten, M. Esposito, and B. Cleuren, Phys. Rev. B 80, 235122 (2009); M. Esposito, R. Kawai, K. Lindenberg, and C. Van den Broeck, Phys. Rev. E 81, 041106 (2010).

[3] M. Esposito, R. Kawai, K. Lindenberg and C. Van den Broeck, Europhys. Lett. 89, 20003 (2010); N. Kumar, C. Van den Broeck, M. Esposito, and K. Lindenberg, Phys. Rev. E 84, $051134(2011)$.

[4] J. Z. He, J. C. Chen, and B. Hua, Phys. Rev. E 65, 036145 (2002).

[5] F. Wu, L. G. Chen, F. R. Sun, C. Wu, and Q. Li, Phys. Rev. E 73, 016103 (2006); F. Wu, L. G. Chen, F. R. Sun, C. Wu, and F. Z. Guo, J. Appl. Phys. 99, 054904 (2006); F. Wu, L. G. Chen, S. Wu, F. R. Sun, and C. Wu, J. Chem. Phys. 124, 214702 (2006).

[6] H. B. Callen, Thermodynamics and an Introduction Thermostatistics (Wiley, New York, 1985), 2nd ed.

[7] A. Bejan, Advanced Engineering Thermodynamics (Wiley, New York, 1997), p. 377.

[8] M. Esposito, R. Kawai, K. Lindenberg, and C. Van den Broeck, Phys. Rev. Lett. 105, 150603 (2010), and references therin.

[9] R. Kosloff, J. Chem. Phys. 80, 1625 (1984).

[10] E. Geva and R. Kosloff, J. Chem. Phys. 96, 3054 (1992); E. Geva and R. Kosloff, J. Chem. Phys. 97, 4396(1992); E. Geva and R. Kosloff, Phys. Rev. E 49, 3903 (1994); E. Geva and R. Kosloff, J. Chem. Phys. 102, 8541 (1995).

[11] T. Feldmann and R. Kosloff Phys. Rev. E 61, 4774 (2000).

[12] Y. Rezek and R. Kosloff, New J. Phys. 8, 83 (2006).

[13] L. Chen and Z. Yan, J. Chem. Phys. 90, 3740 (1989)

[14] C. Van den Broeck, Phys. Rev. Lett. 95, 190602 (2005).

[15] R. S. Berry, V.A. Kazakov, S. Sieniutycz, Z. Szwast, and A. M. Tsvilin, Thermodynamic Optimization of Finite- Time Processes (John Wiley \& Sons, Chichester, 2000).

[16] T. Schmiedl and U. Seifert, Europhys. Lett. 81, 20003 (2008).

[17] Z. C. Tu, J. Phys. A: Math. Theor. 41 (2008); Y. Wang and Z. C. Tu, Phys. Rev. E. 85, 011127 (2012); Y. Wang and Z. C. Tu, arXiv:1110.6493v2.

[18] H. Then and A. Engel, Phys. Rev. E 77, 041105 (2008).

[19] A. Gomez-Marin and J. M. Sancho, Phys. Rev. E 74, 062102 (2006).

[20] Y. Izumida and K. Okuda, Europhys. Lett. 83, 60003 (2008); Phys. Rev. E 80, 021121 (2009); 
Prog. Theor. Phys. Suppl. 178, 163 (2009).

[21] C. M. Bender, D. C. Brody, and B. K. Meister, J. Phys. A: Math. Gen. 33, 4427 (2000).

[22] S. Abe and S. Okuyama, Phys. Rev. E 83, 021121 (2011); S. Abe, Phys. Rev. E 83, 041117 (2011).

[23] J. H. Wang, J. Z. He, and X. He, Phys. Rev. E 84, 041127 (2011); J. H. Wang and J. Z. He, J. Appl. Phys. 111, 043505 (2012).

[24] H. T. Quan, P. Zhang, and C. P. Sun, Phys. Rev. E 73, 036122 (2006); H. T. Quan, Y. X. Liu, C. P. Sun, and F. Nori, Phys. Rev. E 76, 031105 (2007); H. T. Quan, Phys. Rev. E 79, 041129 (2009).

[25] S. W. Kim, T. Sagawa, S. De Liberato, and M. Ueda, Phys. Rev. Lett. 106, 070401 (2011).

[26] J. H. Wang, J. Z. He, and Y. Xin, Phys. Scr. 75, 227 (2007).

[27] M. O. Scully, Phys. Rev. Lett. 104, 207701 (2010); K. E. Dorfman, M. B. Kim, and A. A. Svidzinsky, Phys. Rev. E 84, 053829 (2011).

[28] M. O. Scully, K. R. Chapin, K. E. Dorfman, M. B. Kim, and A. A. Svidzinsky, PNAS 108, 15097 (2011).

[29] M. O. Scully, M. S. Zubairy, G. S. Agarwal, and H. Walther, Science 299, 862 (2003).

[30] H. E. D. Scovil and E. O. Schulz-DuBois, Phys. Rev. Lett. 2, 262 (1959).

[31] K. Nakamura, S. K. Avazbaev, Z. A. Sobirov, D. U. Matrasulov, and T. Monnai, Phys. Rev. E 83, 041133 (2011).

[32] M. Born and V. Fock, Z. Phys. 51, 165 (1928).

[33] For example, we consider a very simple QCE model consisting of an ensemble of many identical noninteracting particles in a one-dimensional power-law potential with size $L$. The power-law potentials can be parameterized by a single-particle energy spectrum of the form $E_{n}(L)=$ $E_{g}(L) n^{\sigma}[23]$, where $E_{g}(L) \equiv E_{1}(L)$ is the energy of the ground state, and $\sigma$ is the index of the single-particle energy spectrum. Without loss of generality, the ground state energy is assumed to be proportional to $L^{-\alpha}$, i.e., $E_{g}(L)=\gamma L^{-\alpha}$, where $\gamma$ is a constant for a given potential, and the index $\alpha$ is positive and depends on the form of the external potential.

[34] For the case with symteric dissipation and without nonadibatic dissipation, in that work [8] the CA efficiency was recovered by optimizating power output with respect to time allocaiton, and thus the correponding time allocation to the hot and cold reservoirs was obtained. 